\title{
The expression of myosin-regulated light chain interacting protein (MYLIP) in lung cancer and its inhibitory effects on lung carcinomas
}

\author{
Wenhui Wang, Fenqiang Li, Peiying Gan, Dongjun Su, Gengxiang Li, Lei Dang, Yuxing Peng \\ Department of Interventional Radiology, Lanzhou University First Affiliated Hospital, Lanzhou, China \\ Contributions: (I) Conception and design: W Wang, F Li, P Gan; (II) Administrative support: D Su, G Li; (III) Provision of study materials or \\ patients: W Wang, L Dang, Y Peng; (IV) Collection and assembly of data: All authors; (V) Data analysis and interpretation: W Wang, F Li, P Gan; (VI) \\ Manuscript writing: All authors; (VII) Final approval of manuscript: All authors. \\ Correspondence to: Wenhui Wang. Department of Interventional Radiology, Lanzhou University First Affiliated Hospital, No. 1 Donggang West \\ Road, Lanzhou 730000, China. Email: wangwenhui1968@126.com.
}

Background: This study explored the relationship between myosin-regulated light chain interacting protein (MYLIP) and the prognosis of lung cancer and its effects on the proliferation, migration, and invasion of lung cancer cells.

Methods: Bioinformatics analyses of databases were conducted to explore the relationship between the expression of MYLIP and the prognosis of lung cancer patients. Real-time fluorescent quantitative polymerase chain reaction and Western blot analyses were used to measure the levels of MYLIP expression. Cell counting kit-8 (CCK8) and cell cloning experiments were used to determine the effects of MYLIP on cell proliferation. The scratch test and invasion experiments were conducted to assess the effects of MYLIP on the migration and invasion of lung cancer cells. Tumor formation experiments were performed in nude mice to determine the effects of MYLIP on tumor growth.

Results: The mRNA and protein expression of MYLIP in cancer tissues from lung cancer patients were significantly lower than that found in normal adjacent tissues $(\mathrm{P}<0.05)$. Bioinformatics analysis showed that lung cancer patients with high MYLIP expression had a better prognosis compared to patients with low MYLIP expression. The results of the CCK8 and cell proliferation experiments revealed that the proliferation ability of lung cancer cells overexpressing MYLIP was significantly lower than that of control cells $(\mathrm{P}<0.05)$. The scratch experiment and invasion experiments demonstrated that the scratch closure rate and the cell invasion ability of lung cancer cells overexpressing Experiments in nude mice showed that the tumor-forming ability of lung cancer cells with high expression of MYLIP was weaker than that of the control group, and the tumor growth rate and the tumor weight were also lower than that of the control group $(\mathrm{P}<0.05)$.

Conclusions: Low levels of MYLIP expression were detected in the cancer tissues of lung cancer patients, and its expression levels were positively correlated with the prognosis of lung cancer. Furthermore, MYLIP had a significant inhibitory effect on the proliferation, migration, and invasion of lung cancer cells, suggesting that MYLIP may be a tumor suppressor gene for lung cancer. The results may have significant potential for clinical applications.

Keywords: Myosin-regulated light chain interacting protein (MYLIP); lung cancer; cell proliferation; cell migration; cell invasion; nude mice model

Submitted Mar 16, 2021. Accepted for publication Apr 29, 2021.

doi: $10.21037 /$ tcr-21-606

View this article at: http://dx.doi.org/10.21037/tcr-21-606 


\section{Introduction}

Lung cancer is the leading cause of death in patients with malignancies. However, there is no clear explanation for the high mortality rate (1). Although lung cancer screening has been extensively implemented in high-risk groups, the overall screening rate is still relatively low. Furthermore, with the worsening of air pollution and the increase in tobacco use, the incidence and mortality of lung cancer in clinical practice continues to increase (2-4). Although there are many treatment options for lung cancer, including surgery, radiotherapy, and chemotherapy, the majority of lung cancer patients are already in the advanced stages at diagnosis and thus treatment is difficult (5). In the past few years, there has been some progress in the targeted therapy of lung cancer. However, this method is not only very expensive, but the effects of the treatment are still controversial (6). There are many pathological types of lung cancer and different subtypes show different tumor incidence, treatment response, and prognosis. Therefore, identifying specific suppressor genes may be important for the development of targeted therapy in lung cancer patients.

Myosin-regulated light chain interacting protein (MYLIP), also known as the ubiquitin-protein ligase, can regulate the post-transcriptional diversity of low-density lipoprotein (LDL) receptors, and can specifically induce the degradation of LDL receptor proteins (7). Studies have shown that MYLIP plays an important role in the development of cancer. For example, MYLIP can inhibit the metastasis and progression of breast cancer $(8,9)$ and inhibit the growth of prostate cancer (10). Some studies have demonstrated that MYLIP can inhibit the progression of lung adenocarcinoma, and the hypoxic environment of the tumor can promote the DNA methylation of MYLIP. This latter study suggested that lung adenocarcinoma patients with low MYLIP methylation or high MYLIP expression had better prognosis (10). Similarly, it is possible that MYLIP acts as a tumor suppressor for lung cancer. Therefore, this study examined the effects of MYLIP on the proliferation, migration, and invasion of lung cancer cells. Understanding the role of MYLIP may provide an informative basis for the diagnosis and development of novel treatments for lung cancer. We present the following article in accordance with the MDAR checklist (available at http://dx.doi.org/10.21037/tcr-21-606).

\section{Methods}

\section{Cell lines and experimental materials}

The human lung cell line Beas-2b and the human lung cancer cell lines including A549, H1299, H460, and H146 were purchased from (FuHeng Cell Center, Shanghai, China). Dulbecco's modified Eagle's medium (DMEM) medium, fetal bovine serum (FBS), and pancreatin were purchased from Gibco, USA. The lentivirus packaging helper plasmid psMYLIP was designed and synthesized by Addgene (Massachusetts, USA). The real-time polyermase chain reaction (PCR) kit was purchased from TAKARA, Japan, and the Cell Counting Kit -8 (CCK8) kit was obtained from Tongren, Japan. The PrimeScript RT kit was purchased from Beijing Baori Biotechnology Co., Ltd. and MYLIP antibody was purchased from Abcam, USA.

\section{Experimental animals}

Heathly 4-week-old male nude mice were provided by the Lanzhou University First Affiliated Hospital Experimental Animal Department and housed in specific pathogen free (SPF) areas with alternating day and night cycles. The temperatures and humidity were suitable for normal breeding. All animal experiments were approved by the animal ethics committee of the Lanzhou University First Affiliated Hospital and all operations complied with the animal experiment regulations.

\section{Tissue samples of lung cancer patients}

Cancerous lung tissues and adjacent normal tissues were obtained from lung cancer patients at the Lanzhou University First Affiliated Hospital. The study conformed to the provisions of the Declaration of Helsinki (as revised in 2013) and study was approved by the Ethics Committee of Lanzhou University First Affiliated Hospital (NO.: LDYYLL2021-193) and informed consent was taken from all the patients.

\section{Experimental methods}

\section{Cell culture}

The normal lung cell line Beas-2b and the lung cancer cell lines, including A549, H1299, H460, and H146, were 
cultured in DMEM medium containing $10 \%$ FBS at $37^{\circ} \mathrm{C}$ with $50 \mathrm{~mL} / \mathrm{L} \mathrm{CO}_{2}$ and saturated humidity. The cells were passaged and used for experiments at $90 \%$ confluency.

\section{Lentivirus infection and verification of MYLIP overexpression}

The A549 and H4601 lung cancer cell lines were inoculated into 6-well plates separately. At 60-70\% confluency, the cells were infected with the lentivirus containing the empty vector or the psMYLIP plasmid, and incubated at $37^{\circ} \mathrm{C}$. Cells were examined under a fluorescence microscope to determine the infection efficiency after $24,48,72$, and 96 hours.

\section{RNA extraction and real-time fluorescence quantitative polymerase chain reaction (PCR)}

RNA from normal lung cells, lung cancer cells, and lung cancer cells overexpressing MYLIP were extracted with RNA extraction reagents according to the manufacturer's instructions. The concentration of RNA was measured and a $1 \mathrm{~L}$ sample was used for the reverse transcription reaction. The corresponding primers, templates, and reagents were then added to the $25 \mathrm{~L}$ system and 40 cycles of the following were performed: pre-denaturation at $94{ }^{\circ} \mathrm{C}$ for 3 minutes, denaturation at $94{ }^{\circ} \mathrm{C}$ for 4 seconds, annealing at $56{ }^{\circ} \mathrm{C}$ for 5 seconds, and extension at $72{ }^{\circ} \mathrm{C}$ for 6 seconds. The forward and reverse primers for MYLIP were 5'-CCGCTGCACATCGTC-3' and 5'-CGTGGAAGGCGGTGATCAG-3', respectively. Glyceraldehyde 3-phosphate dehydrogenase (GAPDH) was used as an internal reference, and the relative expression level of MYLIP was calculated using the formula $2^{-\Delta \Delta C t}$.

\section{Western blot analysis}

Normal lung cells, lung cancer cells, lung cancer cells overexpressing MYLIP, and lung tissues from lung cancer patients were collected. After the lung tissue was homogenized into a single-cell suspension, it was treated with lysis buffer containing protease inhibitors for $30 \mathrm{~min}$ at $4{ }^{\circ} \mathrm{C}$. Samples were then centrifugation at $12,000 \mathrm{rpm}$ for $15 \mathrm{~min}$ at $4{ }^{\circ} \mathrm{C}$, the supernatant was collected, and the protein concentration was determined using the BCA method. All samples were adjusted to the same protein concentration and $20 \mu \mathrm{g}$ was taken for electrophoresis and transferred onto PVDF membranes. The membranes were then blocked with blocking solution containing $5 \%$ skim milk at room temperature for $2 \mathrm{~h}$, followed by incubation with the primary antibody at $4{ }^{\circ} \mathrm{C}$ overnight. The membranes were then washed 3 times with TBST (Tris-buffered saline Tween) for 6 minutes each time. Subsequently, the membranes were incubated with the mouse monoclonal secondary antibody containing horseradish peroxidase at room temperature for 1 hour and washed again with TBST 3 times. The Image J software was used to analyze the band gray value.

\section{Clone formation and CCK8 proliferation experiments} A549 and H460 cells overexpressing MYLIP in the logarithmic growth phase and their corresponding empty vector control cells were harvested and trypsinized into single-cell suspensions. The cells were then diluted in a gradient, and 200 cells were inoculated into preheated $\left(37^{\circ} \mathrm{C}\right) 10 \mathrm{~mL}$ petri dishes, and placed in an incubator for 1-2 weeks. When macroscopic cell clones could be observed in the petri dishes, the supernatant was discarded and the cells were rinsed with phosphate-buffered saline (PBS). The cells were fixed and stained with Giemsa. The cell clones were visualized and counted with the naked eye .

For the CCK8 experiments, A549 and H460 cells overexpressing MYLIP in the logarithmic growth phase and their corresponding empty vector control cells were harvested and trypsinized. After adjusting the cell concentrations, $100 \mathrm{~L}$ of cell solution was inoculated into 96-well plates and cultured at $37{ }^{\circ} \mathrm{C}$ for $0,24,48,72$, and $96 \mathrm{~h}$. Cells were then incubated with the CCK8 solution for 1 hour and the absorbance at $450 \mathrm{~nm}$ was measured with a microplate reader.

\section{Scratch test and in vitro transwell invasion assay}

The A549 and H460 cells overexpressing MYLIP and their corresponding empty vector control cells were cultured in 6-well plates. At $90 \%$ confluency, the head of a cell scraper was used to draw a line in the middle of the cell culture plate. The cell culture plate was rinsed 3 times with PBS and serum-free medium was added. The cells were incubated continuously for $48 \mathrm{~h}$ under normal conditions, and pictures were taken at 0 hours and $48 \mathrm{~h}$ to calculate the scratch closure rate.

For the transwell invasion assays, $100 \mathrm{~L}$ Matrigel (diluted with serum-free medium) was added to the upper chamber of the transwell and air-dried in a $37{ }^{\circ} \mathrm{C}$ incubator. Subsequently, $500 \mathrm{~L}$ medium containing $100 \mathrm{~mL} / \mathrm{L}$ FBS was added to the lower chamber, and $200 \mathrm{~L}$ single-cell suspension $\left(1 \times 10^{5} / \mathrm{mL}\right)$ was added to the upper chamber. After $48 \mathrm{~h}$ of normal culture, cells on the upper layer of the Matrigel were wiped off and cells that had migrated 
through the membrane were stained with Giemsa. Cells were counted from 5 random fields of view under an inverted microscope and pictures were taken.

\section{Tumor formation experiments using nude mice}

A total of 18 4-week-old male nude mice were randomly divided into three groups. A total of $5 \times 10^{6}$ normal lung tissue cells, A546 lung cancer cells, and H460 lung cancer cells were prepared. Mice were anesthetized and the corresponding cells were subcutaneously injected into the back of the mice. Tumor growth was observed and recorded every 3 days, and the experiment was terminated 21 days after inoculation, and the volume and weight of the tumor was measured.

\section{Statistical analysis}

SPSS18.0 software was used to analyze the experimental data. The measurement data were expressed as mean \pm standard deviation, and the comparison between different groups was performed using the $t$-test. A $\mathrm{P}$ value $<0.05$ was considered statistically significant.

\section{Results}

The expression of MYLIP and its relationship with patient prognosis

Analysis using the Kaplan-Meier plotter found that the expression of MYLIP was positively correlated with the prognosis of lung tumor patients. The higher the expression of MYLIP, the better the prognosis of the patient and the two probes were highly consistent (Figure $1 A$ ). The results from the The Cancer Genome Atlas (TCGA) database also revealed that lung cancer patients with high MYLIP expression had better prognosis (Figure 1B). MYLIP expression in lung carcinoma tissue was lower than that in normal adjacent tissues. Furthermore, the MYLIP expression in samples of lung tumor patients at different pathological stages decreased with the increasing degree of lymph node metastasis (Figure 1C,D).

\section{Expression of MYLIP in lung cancer cell lines}

In cell experiments, the level of MYLIP mRNA expression in all the lung cancer cell lines examined was significantly lower than that of normal lung cells (Figure $2 A ; \mathrm{P}<0.05$ ). The level of MYLIP protein was also significantly lower than that of normal lung cells (Figure $2 B, C ; \mathrm{P}<0.05$ ). Lentivirus was used to package A545 and $\mathrm{H} 460$ cells to stably overexpress MYLIP. The mRNA and protein levels of MYLIP were significantly higher in the cells overexpressing MYLIP compared to the corresponding control cells expressing the empty vector (Figure $2 D, E, F ; \mathrm{P}<0.05$ ).

\section{Overexpression of MYLIP inbibits the proliferation of lung cancer cells}

The proliferation ability of the A549 and H460 cell lines overexpressing MYLIP was examined using the CCK8 assay. Cells overexpressing MYLIP had lower proliferation compared with control cells (Figure $3 A, B$ ). In the clone formation experiment, the number of clones in the A549 and H460 cell lines overexpressing MYLIP was significantly less than that of the control cells (Figure $3 C, D, E ; \mathrm{P}<0.05$ ) suggesting that overexpression of MYLIP can inhibit the proliferation of lung cancer cells.

\section{MYLIP inbibits the migration and invasion of lung cancer cells}

The scratch test demonstrated that A549 and H460 cells overexpressing MYLIP had poorer cell migration ability compared to control A549 and H460 cells (Figure 4A,B,C; $\mathrm{P}<0.001)$. In the cell invasion experiment, the number of invasive cells in the MYLIP overexpression group was significantly reduced compared to the control group (Figure $4 D, E, F ; \mathrm{P}<0.001$ ), indicating that MYLIP can inhibit the migration and invasion of lung cancer cells.

\section{MYLIP inhibits tumor formation in vivo}

The A549 and H460 cell lines stably overexpressing MYLIP were inoculated into nude mice. The resultant tumor sizes were significantly smaller than that in mice inoculated with control cells (Figure $5 A, B ; \mathrm{P}<0.001$ ), and the tumor growth rate was also slower than that of the control group (Figure 5C,D; $\mathrm{P}<0.05$ ), indicating that MYLIP had an inhibitory effect on tumor formation.

\section{MYLIP is lowly expressed in lung cancer tissues}

RT-PCR results showed that the level of MYLIP mRNA in lung tumor tissues from patients was significantly lower than that of adjacent normal tissues (Figure 6A). 

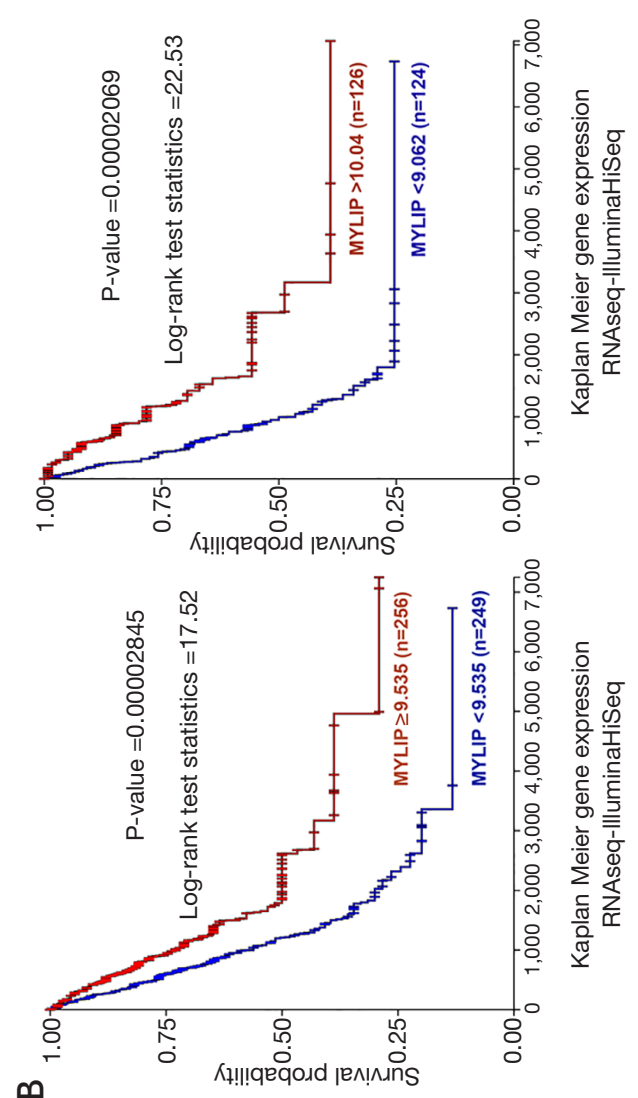

$\infty$
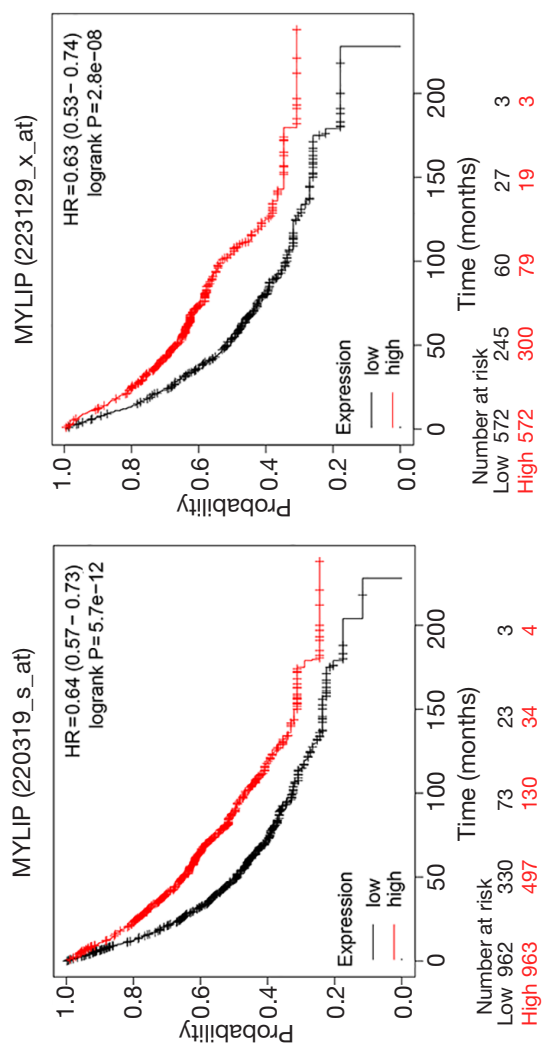

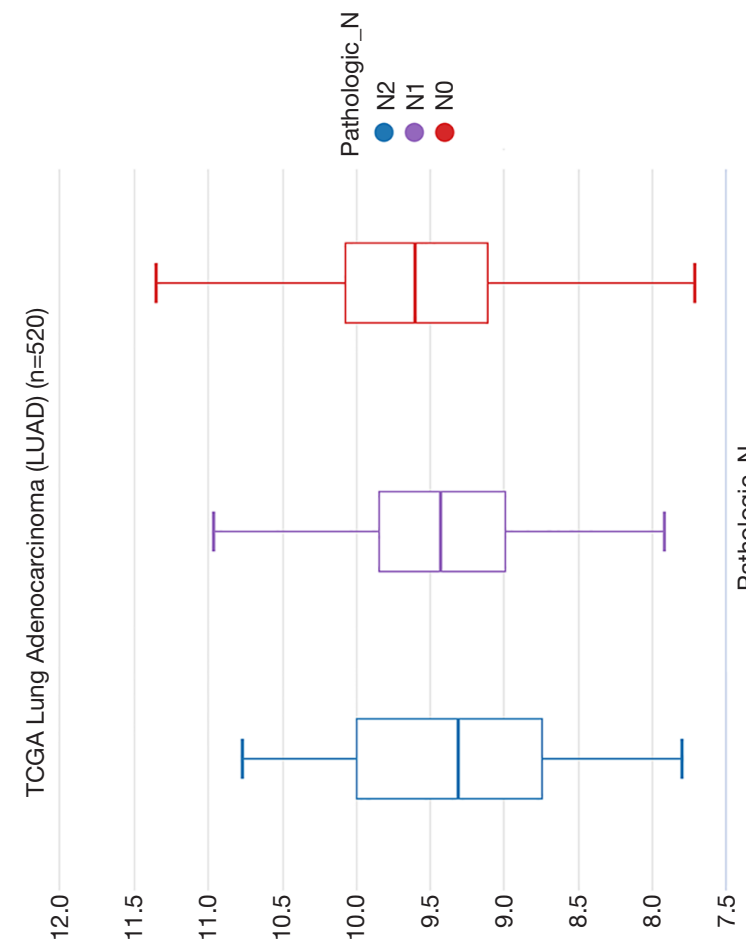

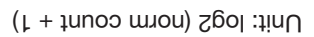

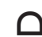
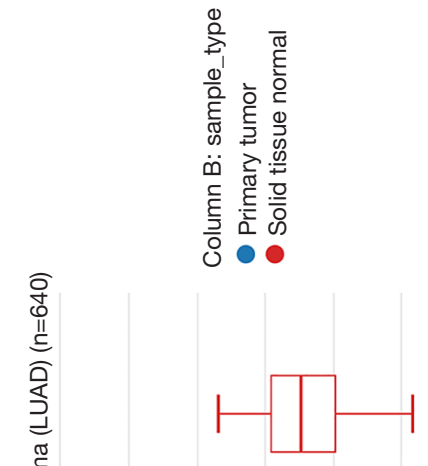

$\cup$

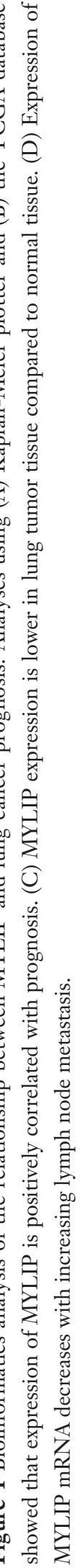



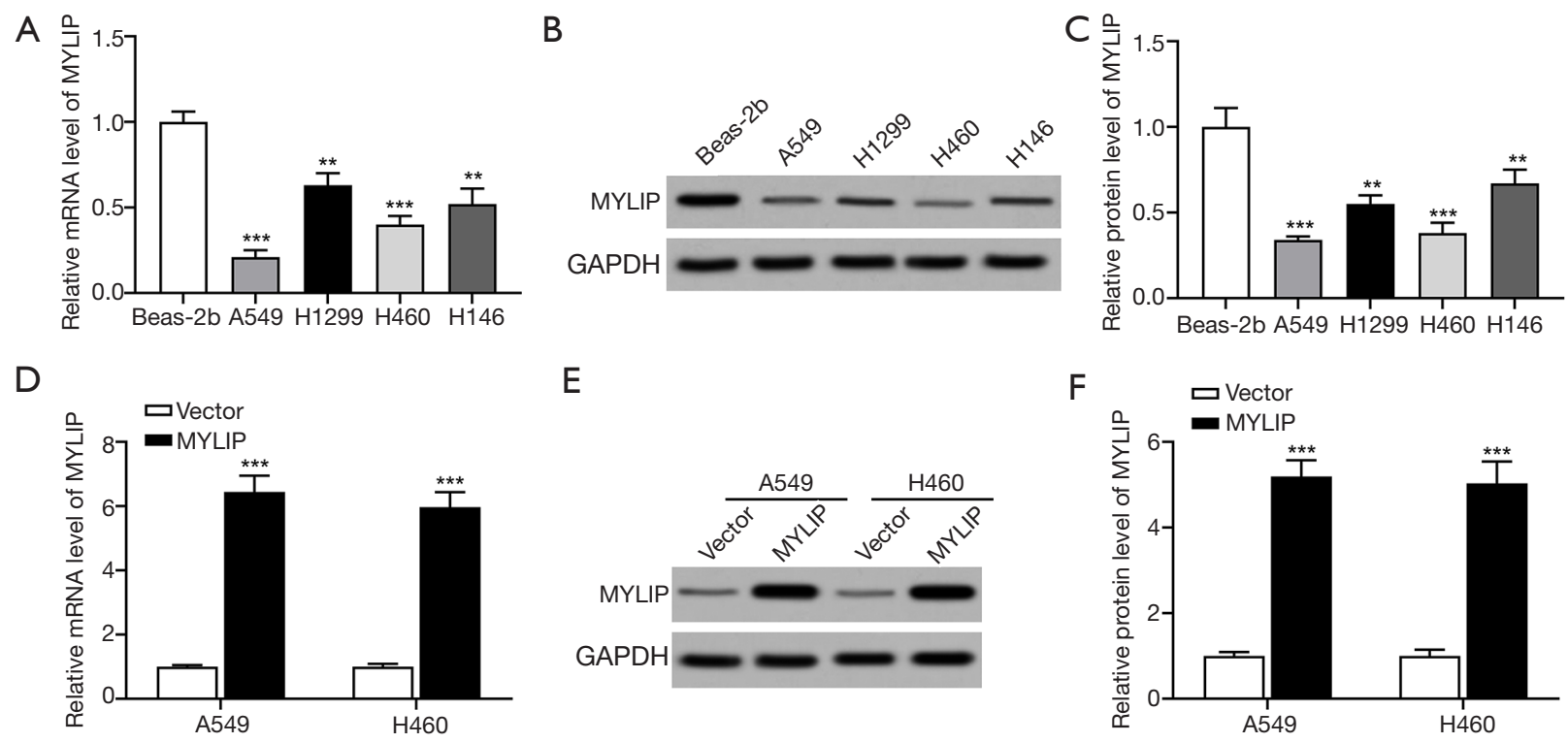

Figure 2 MYLIP mRNA and protein expression in different cell lines. (A) The level of MYLIP mRNA expression in Beas-2b normal lung cells is significantly higher than that in the lung cancer cell lines A549, H1299, H460, and H146. (B) and (C) The protein expression of MYLIP is significantly higher in Beas-2b normal lung cells compared to the lung cancer cell lines A549, H1299, H460, and H146. Overexpression of MYLIP in A549 and H460 cells significantly increased (D) mRNA and (E) protein expression of MYLIP. (F) Relative protein expression of MYLIP in A549 and H460 cells. ${ }^{* *} \mathrm{P}<0.01 ;{ }^{* * *} \mathrm{P}<0.001$.

A

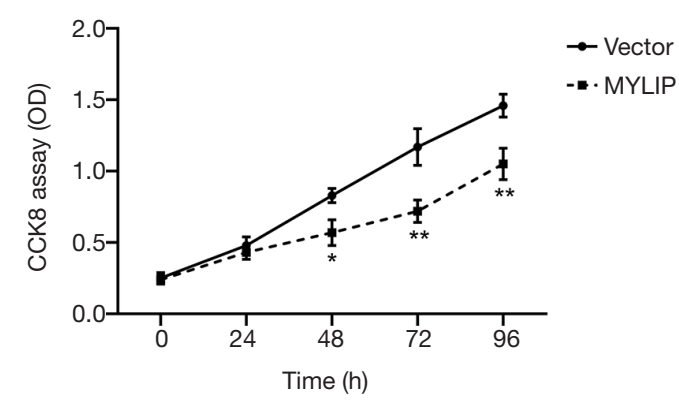

C

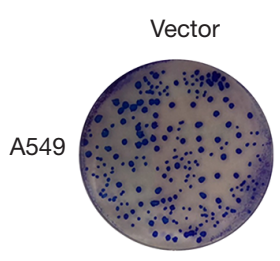

A549

D

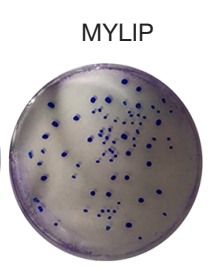

B

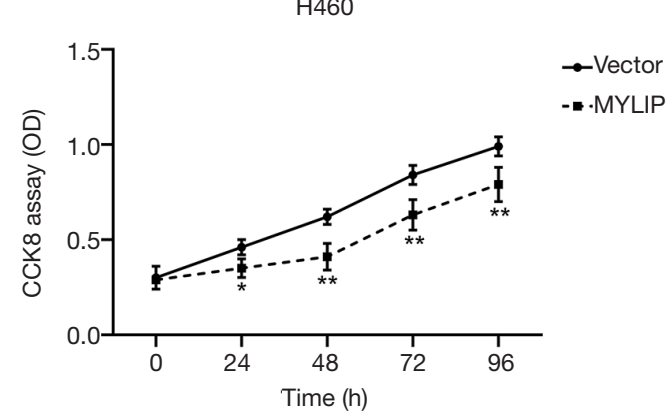

$E$
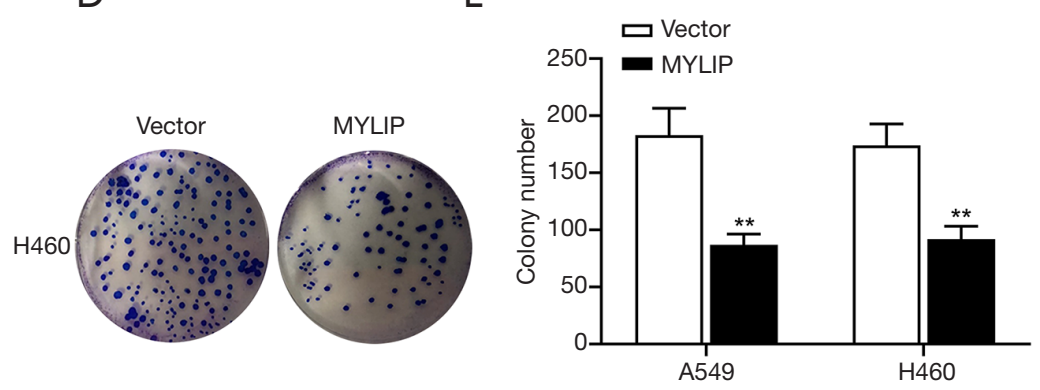

Figure 3 The effects of MYLIP on the proliferation of lung cancer cells. CCK8 assay in (A) A549 and (B) H460 cells shows that overexpression of MYLIP significantly decreased cell proliferation compared to cells transfected with the empty vector. Clone formation assays in (C) A549 and (D) H460 cells shows that overexpression of MYLIP significantly inhibited the ability of cells to form colonies. (E) Graph showing colony formation. ${ }^{*} \mathrm{P}<0.05 ;{ }^{* *} \mathrm{P}<0.01$. 
A
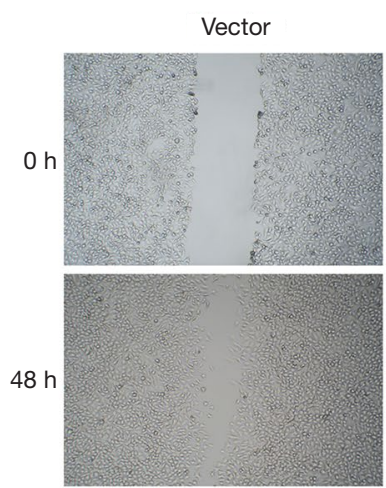

B

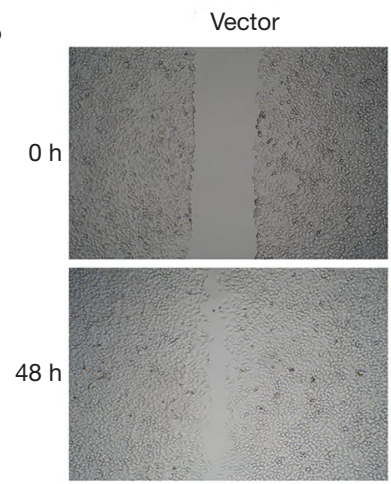

C

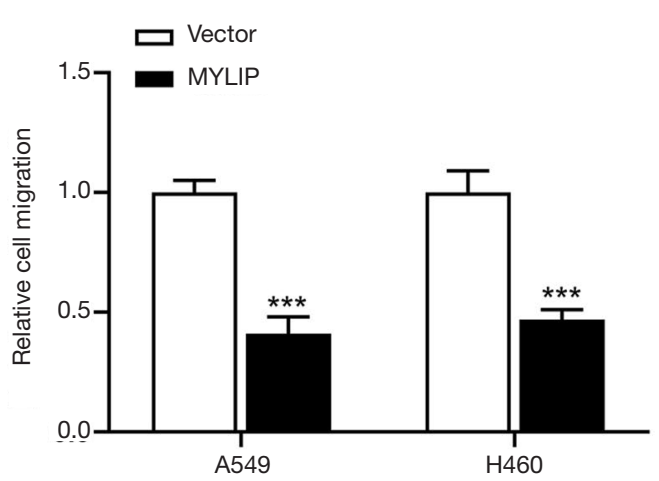

MYLIP

MYLIP
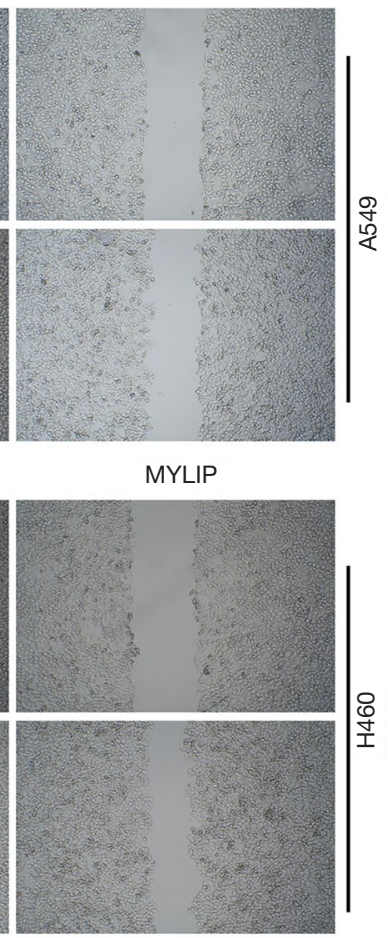

$\mathrm{F}$
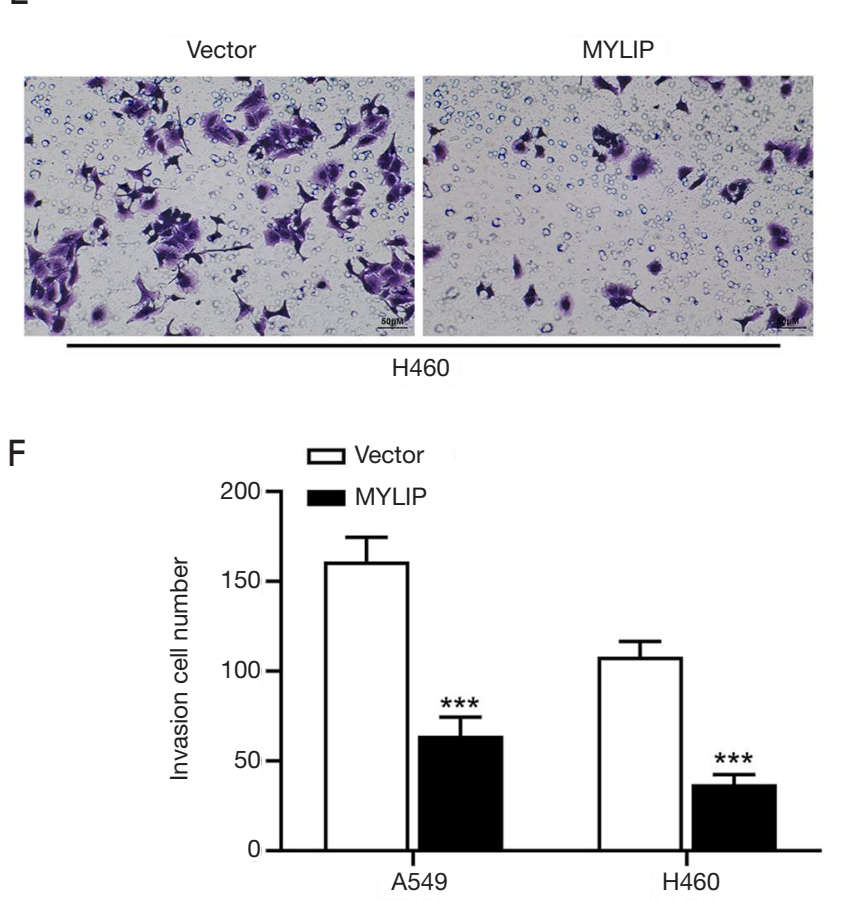

Figure 4 The effects of MYLIP on lung cancer cell migration and invasion. The scratch test in (A) A549 cells and (B) H460 cells shows that overexpression of MYLIP inhibited lung cancer cell migration. magnification, 40x; (C) Graph showing migration ability of cells. In vitro cell invasion assays in (D) A549 cells and (E) H460 cells demonstrate that overexpression of MYLIP inhibited the invasion ability of lung cancer cells. scale bar represents $50 \mu \mathrm{m}$, cells were stained with $0.5 \%$ crystal violet reagent; (F) Graph showing cell invasion ability of lung cancer cells. ${ }^{* * *} \mathrm{P}<0.001$.

Furthermore, Western blot analyses in 20 patients also demonstrated that MYLIP protein levels in many samples were significantly lower than that in the corresponding adjacent tissues (Figure 6B), suggesting that MYLIP is lowly expressed in lung cancer tissues.

\section{Discussion}

MYLIP is a new E3 ubiquitin ligase which can promote the degradation of the myosin-regulated light chain (MRLC) and low-density lipoprotein receptor (LDLR) proteins through the ubiquitin/proteasome pathway (11). Reports 
A

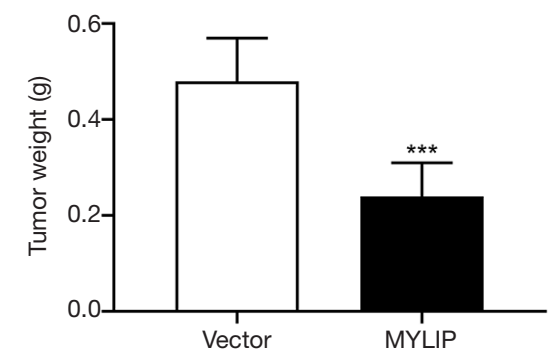

C

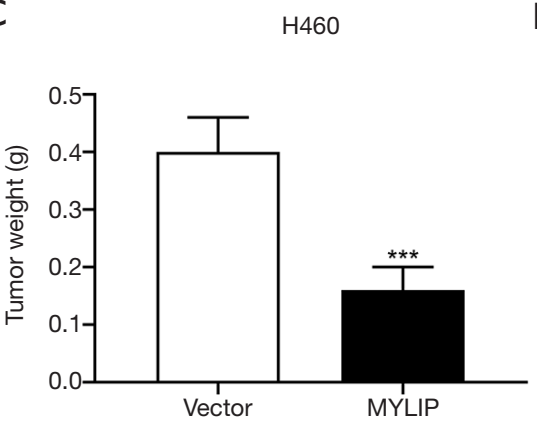

B
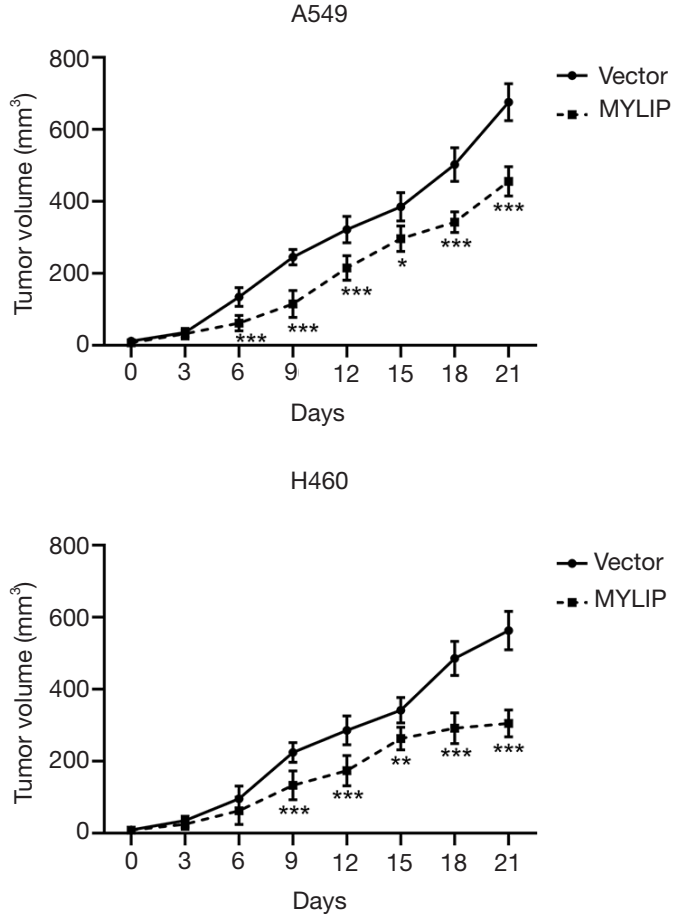

Figure 5 Tumor size and growth in nude mice inoculated with lung cancer cells. The tumor weight in mice inoculated with (A) A549 and (C) H460 cells overexpressing MYLIP was significantly less compared to mice inoculated with cells expressing the empty vector. Similarly, the rate of tumor growth in mice inoculated with (C) A549 and (D) H460 cells overexpressing MYLIP was significantly lower compared to mice inoculated with cells expressing the empty vector. ${ }^{*} \mathrm{P}<0.05 ;{ }^{* *} \mathrm{P}<0.01 ;{ }^{* * *} \mathrm{P}<0.001$.
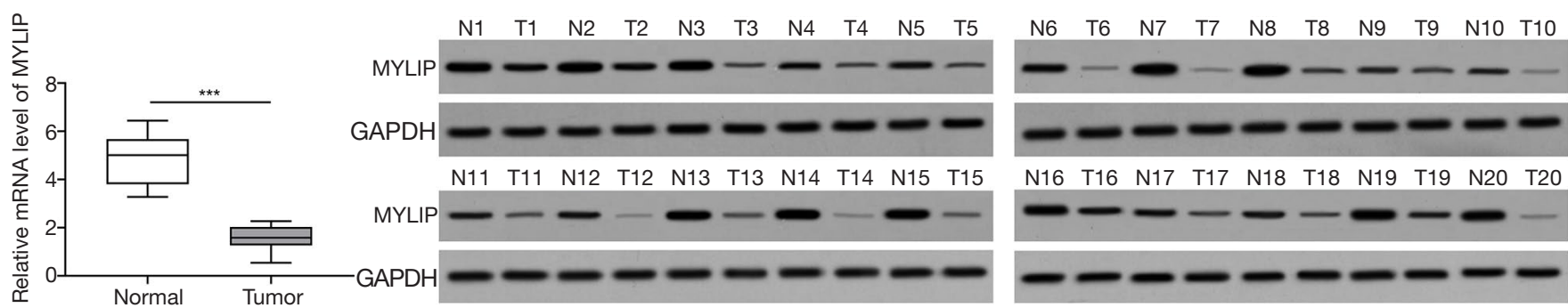

Figure 6 mRNA and protein expression of MYLIP in cancer tissues of lung cancer patients. (A) mRNA expression of MYLIP is significantly lower in lung tumor samples compared to the adjacent healthy tissue. (B) The protein expression of MYLIP was examined in 20 patients with lung cancer. In many samples, lung cancer tissue had lower MYLIP expression compared to the corresponding healthy tissues. ${ }^{* * *} \mathrm{P}<0.001$.

have shown that the MYLIP RING-in-between-RING (RBR) domain can interact with the UBE2D family of ubiquitin-conjugating enzymes (UBE2D1-4) to transfer ubiquitin to the target protein for degradation $(12,13)$. Increasing evidence have demonstrated that E3 ligase plays a crucial role in tumorigenesis and development by targeting the degradation of tumor promoters or suppressors in human malignant tumors $(14,15)$. Various inhibitors of the ubiquitin-proteasome system have been developed, some of which are used as drugs for cancer treatment $(16,17)$. E3 ligase is particularly strict for substrate specificity, thus inhibition of $\mathrm{E} 3$ can be effective for controlling the expression of specific proteins. The LDLR has been shown to be a target substrate of MYLIP (18), although little is 
know about other substrates of MYLIP. Several E3 ligases can interact with their receptors $(19,20)$, but whether MYLIP interacts with its receptors remains to be studied. To date, the relationship between MYLIP and lung cancer has only been reported in one study. The report pointed out that MYLIP is a tumor suppressor in the progression of lung adenocarcinoma. The hypoxic environment of lung cancer promoteds DNA methylation of MYLIP, and suggests that patients with MYLIP hypomethylation or high expression of lung adenocarcinoma have a better prognosis, while tumor growth is subject to complex cellular

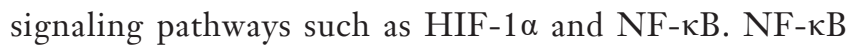
may be directly activated during hypoxia-related lung cancer progression (10). Therefore, studying the function of MYLIP and its mechanisms of action may reveal a novel therapeutic target for lung cancer treatment.

This study demonstrated that the levels of MYLIP expression were reduced in cancer tissues of lung cancer patients. Furthermore, bioinformatics analysis found that patients with high MYLIP expression had better prognosis, suggesting that MYLIP may be a potential tumor suppressor gene for lung cancer. Consistent with this hypothesis, this current study showed that overexpression of MYLIP inhibited the proliferation, migration, and invasion of lung cancer cells in vitro and in vivo using nude mice. In lung cancer cells overexpressing MYLIP, a significant reduction in cell proliferation and the number of cell clones were observed. The cell closure rate and the number of cells invading also decreased, suggesting that MYLIP inhibited the growth of lung cancer cells. Concurrently, in vivo functional experiments using nude mice demonstrated that the rate of tumor formation of lung cancer cells overexpressing MYLIP was slower. The size and weight of the tumor at the conclusion of the experiment were also less than that observed in the control group. This further confirmed that overexpression of MYLIP inhibited lung cancer growth in vivo. Thus, understanding the inhibitory effects of MYLIP on lung cancer may lead to novel promising therapeutic targets for the treatment of lung cancer.

In summary, the low expression level of MYLIP in lung cancer tissues and its inhibitory effects on lung cancer cells suggests that it may be a novel potential target for the treatment of lung cancer. This has important implications for future clinical applications. Futher in-depth studies examining the mechanisms by which MYLIP inhibits lung cancer will provide a theoretical basis for future targeted therapies.

\section{Acknowledgments}

Funding: This work was supported by the Gansu Province Technology Research and Development Special Plan Project (1207TCYA018).

\section{Footnote}

Reporting Checklist: The authors have completed the MDAR checklist. Available at http://dx.doi.org/10.21037/tcr-21-606

Data Sharing Statement: Available at http://dx.doi. org/10.21037/tcr-21-606

Conflicts of Interest: All authors have completed the ICMJE uniform disclosure form (available at http://dx.doi. org/10.21037/tcr-21-606). The authors have no conflicts of interest to declare.

Etbical Statement: The authors are accountable for all aspects of the work in ensuring that questions related to the accuracy or integrity of any part of the work are appropriately investigated and resolved. The study conformed to the provisions of the Declaration of Helsinki (as revised in 2013) and was approved by the Ethics Committee of Lanzhou University First Affiliated Hospital (NO.: LDYYLL2021-193) and informed consent was taken from all the patients. All animal experiments were approved by the animal ethics committee of the Lanzhou University First Affiliated Hospital and all operations complied with the animal experiment regulations.

Open Access Statement: This is an Open Access article distributed in accordance with the Creative Commons Attribution-NonCommercial-NoDerivs 4.0 International License (CC BY-NC-ND 4.0), which permits the noncommercial replication and distribution of the article with the strict proviso that no changes or edits are made and the original work is properly cited (including links to both the formal publication through the relevant DOI and the license). See: https://creativecommons.org/licenses/by-nc-nd/4.0/.

\section{References}

1. Romaszko AM, Doboszyńska A. Multiple primary lung cancer: A literature review. Adv Clin Exp Med 2018;27:725-30.

2. Torre LA, Bray F, Siegel RL, et al. Global cancer statistics, 
2012. CA Cancer J Clin 2015;65:87-108.

3. Feng RM, Zong YN, Cao SM, et al. Current cancer situation in China: good or bad news from the 2018 Global Cancer Statistics?. Cancer Commun (Lond) 2019;39:22.

4. Bray F, Ferlay J, Soerjomataram I, et al. Global cancer statistics 2018: GLOBOCAN estimates of incidence and mortality worldwide for 36 cancers in 185 countries. CA Cancer J Clin 2018;68:394-424. Erratum in: CA Cancer J Clin 2020;70:313.

5. Jin D, Guo J, Wu Y, et al. Metformin-repressed miR381-YAP-snail axis activity disrupts NSCLC growth and metastasis. J Exp Clin Cancer Res 2020;39:6.

6. The Lancet. Lung cancer: some progress, but still a lot more to do. Lancet 2019;394:1880.

7. Nelson JK, Sorrentino V, Avagliano Trezza R, et al. The Deubiquitylase USP2 Regulates the LDLR Pathway by Counteracting the E3-Ubiquitin Ligase IDOL. Circ Res 2016;118:410-19.

8. Zhao L, Zhao Y, He Y, et al. miR-19b promotes breast cancer metastasis through targeting MYLIP and its related cell adhesion molecules. Oncotarget 2017;8:64330-43.

9. Zhao L, Zhou Y, Zhao Y, et al. Long non-coding RNA TUSC8 inhibits breast cancer growth and metastasis via miR-190b-5p/MYLIP axis. Aging (Albany NY) 2020;12:2974-91.

10. Ito S, Ueno A, Ueda T, et al. CNPY2 inhibits MYLIPmediated AR protein degradation in prostate cancer cells. Oncotarget 2018;9:17645-55.

11. Lindholm D, Bornhauser BC, Korhonen L. Mylip makes an Idol turn into regulation of LDL receptor. Cell Mol Life Sci 2009;66:3399-402.

Cite this article as: Wang W, Li F, Gan P, Su D, Li G, Dang L, Peng Y. The expression of myosin-regulated light chain interacting protein (MYLIP) in lung cancer and its inhibitory effects on lung carcinomas. Transl Cancer Res 2021;10(5):23892398. doi: 10.21037/tcr-21-606
12. Wang P, Dai X, Jiang W, et al. RBR E3 ubiquitin ligases in tumorigenesis. Semin Cancer Biol 2020;67:131-144.

13. Zhang L, Fairall L, Goult BT, et al. The IDOL-UBE2D complex mediates sterol-dependent degradation of the LDL receptor. Genes Dev 2011;25:1262-74.

14. Liu J, Peng Y, Zhang J, et al. Targeting SCF E3 Ligases for Cancer Therapies. Adv Exp Med Biol 2020;1217:123-46.

15. Cheng J, Guo J, North BJ, et al. The emerging role for Cullin 4 family of E3 ligases in tumorigenesis. Biochim Biophys Acta Rev Cancer 2019;1871:138-59.

16. Jia X, Li C, Li L, et al. Neddylation Inactivation Facilitates FOXO3a Nuclear Export to Suppress Estrogen Receptor Transcription and Improve Fulvestrant Sensitivity. Clin Cancer Res 2019;25:3658-72.

17. Jin Y, Zhang P, Wang Y, et al. Neddylation Blockade Diminishes Hepatic Metastasis by Dampening Cancer Stem-Like Cells and Angiogenesis in Uveal Melanoma. Clin Cancer Res 2018;24:3741-54.

18. Zelcer N, Hong C, Boyadjian R, et al. LXR regulates cholesterol uptake through Idol-dependent ubiquitination of the LDL receptor. Science 2009;325:100-4.

19. Savoy RM, Chen L, Siddiqui S, et al. Transcription of Nrdp1 by the androgen receptor is regulated by nuclear filamin A in prostate cancer. Endocr Relat Cancer 2015;22:369-86.

20. Li B, Lu W, Chen Z. Regulation of Androgen Receptor by E3 Ubiquitin Ligases: for More or Less. Receptors Clin Investig 2014;1:10.14800\%2Frci.122.

(English Language Editor: J. Teoh) 